\title{
TUNABLE FILTER FOR HIGH MOLECULAR WEIGHT DNA SELECTION AND LINKED-READ SEQUENCING
}

Nicolas MILON ${ }^{1,2}$, Juan-Luis Fuentes Rojas ${ }^{1}$, Adrien Castinel $^{4}$, Laurent Bigot ${ }^{3}$, Géraud Bouwmans ${ }^{3}$, Karen

Baudelle $^{3}$, Audrey Boutonnet ${ }^{2}$, Audrey Gibert ${ }^{4}$, Olivier Bouchez ${ }^{4}$, Cécile Donnadieu ${ }^{4}$, Frédéric Ginot ${ }^{2}$, Aurélien Bancaud $^{1, *}$

${ }^{1}$ CNRS, LAAS, 7 avenue du colonel Roche, F-31400, Toulouse, France.

${ }^{2}$ Adelis Technologies, 478 Rue de la Découverte, 31670 Labège, France

${ }^{3}$ Univ. Lille, CNRS, UMR 8523 - PhLAM - Physique des Lasers Atomes et Molécules, F-59000 Lille, France

${ }^{4}$ INRA, US 1426 GeT-PlaGe, INRA Auzeville, F-31326, Castanet-Tolosan Cedex, France

Correspondence should be sent to Aurélien BANCAUD (abancaud@laas.fr, +33 5613362 46) 


\section{Abstract (148 words)}

In third generation sequencing, the production of quality data requires the selection of molecules longer than $\sim 20 \mathrm{kbp}$, but the size selection threshold of most purification technologies is smaller than this target. Here, we describe a technology operated in a capillary with an accordable selection threshold in the range of 3 to $40 \mathrm{kbp}$ controlled by an electric field. We demonstrate that the selection cut-off is sharp, the purification yield is high, an analytical model predicts the actuation settings of the filter, and the purification throughput is scalable. The selection of high molecular weight genomic DNA from the Melon Cucumis melo L., a diploid organism of $\sim 0.45$ $\mathrm{Gbp}$, is then reported. Linked-read sequencing data show that the N50 phase block size, which scores the correct representation of the two chromosomes, is enhanced by a factor of 2 after size selection, establishing the relevance and versatility of our technology. 


\section{Introduction}

DNA sequencing technologies have been maturing rapidly to enhance the sequencing speed, throughput and associated cost. The list of annotated genomes has been growing in consequence together with the resolution of complex genomic structures ${ }^{1,2}$. Although essential results for research and diagnostics are collected with second generation sequencing technologies (SGS), short-read sequencing holds some intrinsic limitation for the unbiased assembly of genomes ${ }^{3,4}$. The long read capability of third generation sequencing technologies (TGS) ${ }^{5,6}$ overcomes some of these limitations, yet the full potential of TGS relies on quality library preparation with high molecular weight (MW) DNA molecules in the range of 20 to $200 \mathrm{kbp}{ }^{7}$.

The process flow for DNA sequencing starts with a purification step (Fig. 1A), which is most commonly operated by controlling the binding of genomic DNA on a matrix and subsequently releasing it to an appropriate buffer ${ }^{8}$, or by DNA precipitation. Matrix binding and precipitation protocols have performed equally well for the production of quality sequencing data with SGS ${ }^{9}$. However, molecules of more than $\sim 20$ $\mathrm{kbp}$ tend to be degraded during these purification protocols ${ }^{10}$, and a size selection step is usually carried out to remove the low MW residues before library preparation in TGS. If the MW of these by-products is very low, typically a few nucleotides, they can readily eliminated by solid-phase reversible immobilization ${ }^{11,12}$. The selection of higher MW residues is however more laborious, because it is often performed by pulsed-field gel electrophoresis for separation followed by band excision and electroelution ${ }^{13-15}$. Furthermore, the yield of this size selection process is lower than $50 \%$ for molecules of 20 to $50 \mathrm{kbp}$, imposing the purification of large initial amounts of genomic material, which are not always accessible for e.g. single cell sequencing studies ${ }^{16}$. The development of fast and high-yield size selection technologies is therefore direly needed to enhance and speed up the extraction of quality data in TGS.

Microfluidic technologies offer unique solutions for the manipulation and purification of high MW DNA. For instance, DNA electrophoresis in artificial separation matrices made out of periodic arrays of obstacles etched in glass or silicon demonstrated their relevance for the sorting of $\sim 100 \mathrm{kbp}$ in $15 \mathrm{~s}{ }^{17}$. Because DNA molecules are not entrapped in the separation matrix, they can subsequently be sorted and purified by size, as recently reported with a selection cut-off of $\sim 2 \mathrm{kbp}{ }^{18}$. Alternatively, we recently developed the " $\mu$ Laboratory for DNA Analysis and Separation" ( $\mu$ LAS) technology for DNA analysis in the size range 0.1 to $200 \mathrm{kbp}{ }^{19,20}$. This technology is operated in a capillary electrophoresis system by controlling the fluid flow and using a counter electrophoretic force ${ }^{21}$. The key component of $\mu$ LAS is a constriction, in which DNA can be 
concentrated before its analysis with a limit of detection of $10 \mathrm{fg} / \mu \mathrm{L}^{19,20,22}$. Because the technology is operated without separation matrices, it can be used for DNA size selection ${ }^{20}$. Here, we set out the development of a tunable size selection filter to rapidly produce 10 to $20 \mathrm{ng}$ of DNA for TGS. The first section of this report describes the operating principle of this filter, which is subsequently operated in a monocapillary system. The selection cut-off can be tuned with excellent precision in the size range 3 to $40 \mathrm{kbp}$ by monitoring the electric field in the range of 3 to $7 \mathrm{kV} / \mathrm{m}$. We then prove that the throughput of the filter is scalable by parallelizing the technology in a multicapillary system of 61 channels. These settings allow us to produce $17 \mathrm{ng}$ of high MW genomic DNA in two hours. The resulting sample is analyzed by linked-read sequencing, which consists in partitioning long DNA molecules into one million droplets each containing a specific barcode and the material for library preparation, before standard short-read sequencing ${ }^{23}$. Sequencing data are significantly improved with the purification than without it for haplotype resolution. We finally discuss the different applications for which $\mu$ LAS size selection technology may offer advantages to obtain quality sequence data.

\section{Principle of the DNA filter controlled by an electric field}

In the conventional operation of $\mu \mathrm{LAS}{ }^{19,21}$, DNA manipulation is performed in a fluidic system formed by the assembly of two capillaries of different inner diameters of 330 and $50 \mu \mathrm{m}$ (Fig. 1B). The sample is conveyed in the large capillary using a constant pressure source and a counter electrophoretic force (blue and red arrows in Fig. 1B, respectively). In a viscoelastic solution, the shearing of the fluid around DNA molecules is associated to a build-up in normal stress, which leads to migration in the direction transverse to that of the flow. Transverse viscoelastic forces $F_{V E}$ drag and trap DNA molecules at the vicinity of the walls ${ }^{24,25}$ where the flow velocity is low. Because transverse viscoelastic forces are low in the large capillary, hydrodynamic transport is prevalent upstream of the constriction. Conversely, the fast flow velocity in the narrow capillary triggers high transverse viscoelastic forces associated to a mode of transport dominated by electrophoresis, and defining a region of accumulation at the constriction (green rectangle in Fig. 1B). As soon as the electric field decreases, transverse viscoelastic forces decrease and DNA molecules progressively escape from the accumulation region by migrating in the small capillary. The DNA filter exploits the size dependence of viscoelastic transverse forces and consists in setting the electric field to a threshold value that enables the passage of molecules up to a threshold size $N_{c}$ while retaining the high MW fraction (middle panel in Fig. 1B). 
The purified genomic material is finally collected by turning off the electric field and collecting the molecules in a fresh vial (lower panel in Fig. 1B).
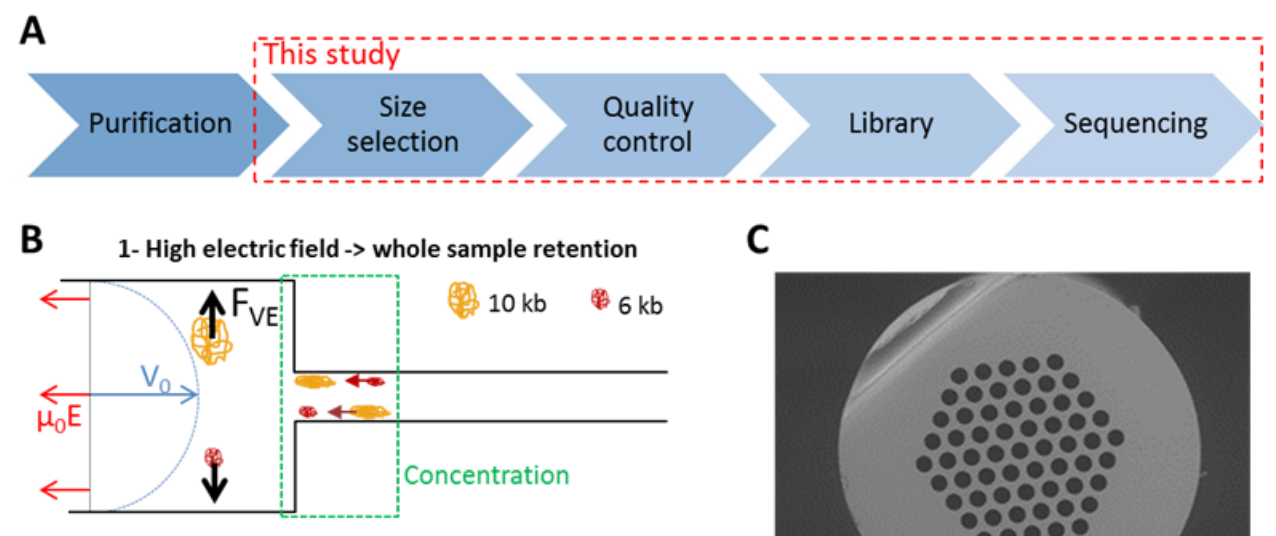

2- Low electric field -> high MW retention, low MW collection
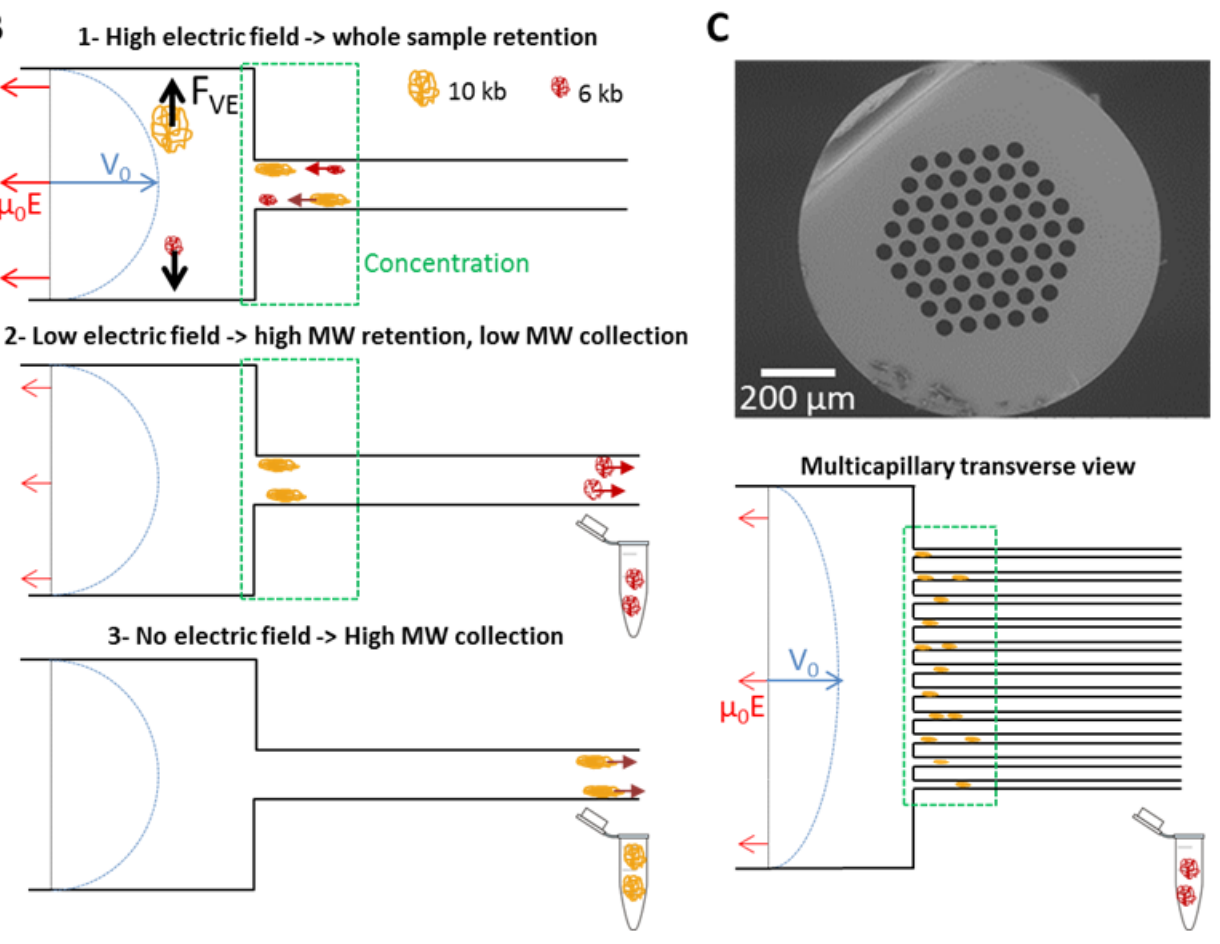

Figure 1 : Principle of the DNA size selection filter for TGS. (A) The panel shows the main steps of the process flow for TGS. Our study is focused on the size selection of genomic DNA and the analysis of the resulting material by linked-read sequencing (see Methods in Supplementary Material). (B) The sketch represents the response of DNA in a capillary system. Transport is controlled by a Poiseuille flow opposed to an electrophoretic force (blue and red arrows, respectively). The balance between hydrodynamic and electrophoretic forces is favorable to hydrodynamic upstream of the constriction and to electrophoresis downstream, defining a region of DNA accumulation represented in green. By tuning the electric field (middle panel), we allow the leak of low MW DNA molecules whereas longer ones remain trapped in the concentrator. The high MW fraction is collected by switching off the electric field (bottom panel). (C) The electron micrograph shows a multicapillary system with 61 channels of $46 \mu \mathrm{m}$, and the sketch presents the processing of DNA in this system. Photographs of the devices shown in panel $(B)$ and $(C)$ are shown in Supplementary Fig. SXX.

More quantitatively, we showed that the transverse viscoelastic force $F_{V E}$ increased linearly with the fluid maximum velocity $V_{0}$ and the electrophoretic velocity $V_{e}=\mu_{0} E$ with $\mu_{0}$ the mobility ${ }^{24,25}$, following a scaling in the form: 


$$
F_{V E}(\varepsilon) \sim N \mathrm{~V}_{0} \mathrm{~V}_{\mathrm{e}} \frac{\varepsilon}{\mathrm{R}}
$$

with $N$ the size of the chain, $R$ the radius of the capillary, and $\varepsilon$ the distance from the walls. The linear dependence of the transverse force with DNA size comes from the fact that the molecule can partitioned into a series of "Kuhn" segments, each undergoing a "monomeric" transverse force and each contributing additively to the total transverse force acting on the molecule. We note that transverse migration also occurs in Newtonian fluids ${ }^{26,27}$, and the transverse force is expected to scale linearly in $V_{0}$ and $V_{e}$ but not in DNA size.

The force in Eq. (1) is equivalent to an elastic spring that keeps the molecule near the wall, allowing us to deduce the average position of DNA from the wall based on Boltzmann statistics ${ }^{24,25}$ :

$$
\langle\varepsilon\rangle \cong \sqrt{\frac{k_{B} T R^{2}}{N V_{0} \mu_{0} E}}
$$

The threshold size $N_{c}$ corresponds to the situation of null velocity with balanced hydrodynamic and electrophoretic velocities at the constriction:

$$
\frac{2 \mathrm{~V}_{0}}{\mathrm{R}}\langle\varepsilon\rangle=\mu_{0} E
$$

Plugging Eq. (2) into Eq. (3), we deduce that:

$$
N_{c} \cong \frac{k_{B} T}{\mu_{0}{ }^{3}} \times \frac{V_{0}}{E^{3}}
$$

Because the electric field can be tuned with excellent precision, the size selection threshold $N_{c}$ appears to be highly accordable. The cubic dependence in electric field also suggests that $N_{c}$ can be adjusted over a broad range with small variations of the electric field.

The prediction of the size selection threshold (Eq. (4)) is expected to be relevant for a multicapillary system, which consists of parallel channels of equal diameter (Fig. 1C, see Methods in Supplementary Material for details on the fabrication protocol). Indeed, the flow velocity and electric field are the same in each narrow channel, and they can be set to the same regime of concentration as in the monocapillary system (green rectangle in Fig. 1C). Notably, because the total flow rate is the sum of that flowing in each channel, we expect the multicapillary system to be a scalable technology for processing larger volumes and larger DNA quantities. 


\section{Experimental}

\section{Reagents}

Molecular biology grades chemicals were purchased from Sigma-Aldrich (St. Louis, MO). The QuickLoad ( $1 \mathrm{~kb}$ Extend DNA Ladder (\# N3239S), containing 13 bands from 0.5 to $50 \mathrm{kbp}$, was purchased from New England Biolabs (Ipswich, MA). Running buffers was composed of 1X Tris-Borate-EDTA (TBE, 89 $\mathrm{mM}$ Tris, $89 \mathrm{mM}$ boric acid, $2 \mathrm{mM}$ EDTA) supplemented with $3 \% 43 \mathrm{kDa}$ PVP ${ }^{19,28}$. The intercalating dye (Picometrics Technologies, France, \# 16-BB-DNA1K/01) was used at a $2 \mathrm{X}$ final concentration in the buffer solution. All buffer solutions were filtered at $0.22 \mu \mathrm{m}$ before use.

\section{Mono and multicapillary systems fabrication}

Fused silica monocapillaries were purchased from Polymicro Technologies (Phoenix, US). The monocapillary device contained three pieces of capillary, (i) $5 \mathrm{~cm}$ in length and $50 \mu \mathrm{m}$ in inner diameter (ID), (ii) $4 \mathrm{~cm}$ in length and $330 \mu \mathrm{m}$ in ID used as an injection chamber of $3.4 \mu \mathrm{L}$, and (iii) $19 \mathrm{~cm}$ in length and 50 $\mu \mathrm{m}$ in ID. Monocapillary devices were assembled manually at Picometrics using a UV-curable acrylate-based glue, as previously described in ${ }^{19}$.

The multi-capillary system is based on the technology developed to manufacture photonic crystal optical fibers by the stack-and-draw method ${ }^{29}$. More precisely, a meter-long silica tube of $25 \mathrm{~mm}$ outer diameter (OD) and $15 \mathrm{~mm}$ ID was elongated into several tens of millimeter-sized capillaries. This operation was performed on a drawing tower, a vertical equipment consisting in a feeding preform unit, a high temperature furnace, and a tractor unit. The resulting monocapillaries were then assembled manually to form a hexagonal stack inserted into a tube of $25 \mathrm{~mm}$ OD and $19 \mathrm{~mm}$ ID. This stack was eventually drawn into a multi-capillary cane using the same drawing tower. A fiber of $\sim 900 \mu \mathrm{m}$ in OD and constituted of 61 capillaries of $45.8 \mu \mathrm{m}$ in diameter (standard deviation $0.6 \mu \mathrm{m}$ ) was obtained. In order to fabricate the multicapillary system, we fitted a $2 \mathrm{~cm}$ long section of multicapillary fiber into a glass vial insert of $300 \mu \mathrm{L}$ (\# $4025 \mathrm{GF}-625$, J.G. Finneran Associates, Vineland, NJ) and assembled it with the acrylate UV-curable glue. 


\section{Instrumentation of capillary systems}

Monocapillary experiments were carried out with an Agilent 1600CE system equipped with a Zetalif LED $480 \mathrm{~nm}$ detector (Picometrics Technologies, France) and modified to install the capillary device and place the optical head of the detector $7 \mathrm{~cm}$ downstream of the concentration junction. Prior to use, every capillary device was rinsed with $1 \mathrm{M} \mathrm{NaOH}$, then with $0.1 \mathrm{M} \mathrm{HCl}$, and finally flushed with de-ionized water. Prior to each experiment, the devices were conditioned with $0.1 \mathrm{M} \mathrm{HCl}, 1 \%$ poly-vinylalcool (PVA), and flushed with running buffer. We used the constriction facing the short capillary of $5 \mathrm{~cm}$ or the long one of $19 \mathrm{~cm}$ for purification or size characterization operations, respectively. This choice was motivated by the need to speed up the flushing of low MW residues during purification operations.

The multicapillary device was operated on a custom prototype with two electrodes connected to the insert and collection vial. Liquid flows characterized by a mean flow velocity of $1.0 \mathrm{~mm} / \mathrm{s}$ were controlled by gravity (See supplementary for hydrodynamic modelling). The samples were manually loaded at the entrance of the fiber and a carousel with several tubes was placed at the outlet for sample fractionation.

After size selection operations with the mono- or multicapillary systems, the different sample fractions were characterized with the $\mu$ LAS separation and titration protocol described in ${ }^{28}$. Sizing and quantification errors are $3 \%$ and $10 \%$, respectively.

\section{Sequencing library construction using the GemCode platform}

A Chromium Controller Instrument (10x Genomics) was used for sample preparation at the GeTPlaGe core facility (Toulouse, France). Sample indexing and partition barcoded libraries were prepared using the Chromium Genome Sequencing Solution (10x Genomics, Pleasanton, CA). GEM reactions were performed on $5 \mathrm{ng}$ of genomic DNA, with or without $\mu$ LAS size selection, and DNA molecules were partitioned and amplified into droplets to introduce a 16-bp partition barcodes. GEM reactions were thermally cycled $\left(30^{\circ} \mathrm{C}\right.$ for $3 \mathrm{~h}$ and $65^{\circ} \mathrm{C}$ for $10 \mathrm{~min}$; held at $\left.4^{\circ} \mathrm{C}\right)$ and after amplification the droplets were fractured. P5 and P7 primers, Read 2, and Sample Index were added during library construction according to 10x Genomics protocol. Libraries were amplified using 10 cycles of PCR and the DNA was subsequently size selected to 450 bp by performing a double purification on AMPure Xp beads. Library quality was 
assessed using a Fragment Analyzer and quantified by qPCR using the Kapa Library Quantification Kit. Sequencing was conducted with an Illumina Hiseq3000 with $2 \times 151$ paired-end reads based on the manufacturer's protocols. The depth of sequencing was $65 \mathrm{X}$.

\section{Alignment, barcode assignment and calculation of sequencing metrics}

The LongRanger analysis software (10x Genomics, v2.2.2) was used to convert sequences to fastQ files and to remove sequences that do not have the exact index sequence given by Chromium. The fastQ files in output were then used to produce a graph-based assembly by Supernova (10x Genomics, v2.2.2). The Fasta file of genome assembly was created with pseudohap formatting option. Finally, statistics were computed by Quast software (quast-5.0). Analysis was performed thanks to the Genotoul bioinformatics platform in Toulouse, Midi-Pyrenees (Bioinfo Genotoul), which provided computing and storage resources.

\section{Results}

\section{DNA size selection in a monocapillary system}

We first characterized the size selection filter with a high MW DNA ladder containing 13 bands between 0.5 to $50 \mathrm{kbp}$ and using a commercial capillary electrophoresis system (see Methods). Our experiments consisted in loading $0.1 \mathrm{ng}$ of DNA ladder in $1 \mu \mathrm{L}$, i.e. at a concentration of $100 \mathrm{pg} / \mu \mathrm{L}$, in the monocapillary system (upper panel of Fig. 1B). During a first phase of 60 minutes, we eliminated the low MW fraction of the sample with a flow of buffer at a velocity of $\sim 1 \mathrm{~mm} / \mathrm{s}$ and different settings for the electric field spanning 3 to 7 $\mathrm{kV} / \mathrm{m}$ (middle panel in Fig. 1B). The length of the small capillary was $5 \mathrm{~cm}$, so the convection time of the buffer was one minute, i.e. much less than the process time of 60 minutes. In fact, the duration of this step was adjusted to ensure the elimination of low MW molecules, which migrate at a slow velocity compared to the buffer due to transverse forces. For instance, the typical migration speed of a $20 \mathrm{kbp}$ fragment was $\sim 0.13$ $\mathrm{cm} / \mathrm{min}$ (data not shown), so that $\sim 40 \mathrm{~min}$ were necessary for their escape from the $5 \mathrm{~cm}$-long capillary. The 
volume of fluid collected at the outlet during these 60 minutes was $\sim 10 \mu \mathrm{L}$, and since it was collected in a fresh vial initially filled with $20 \mu \mathrm{L}$ of buffer, the dilution of the low MW "leak" fraction was 30-fold. 

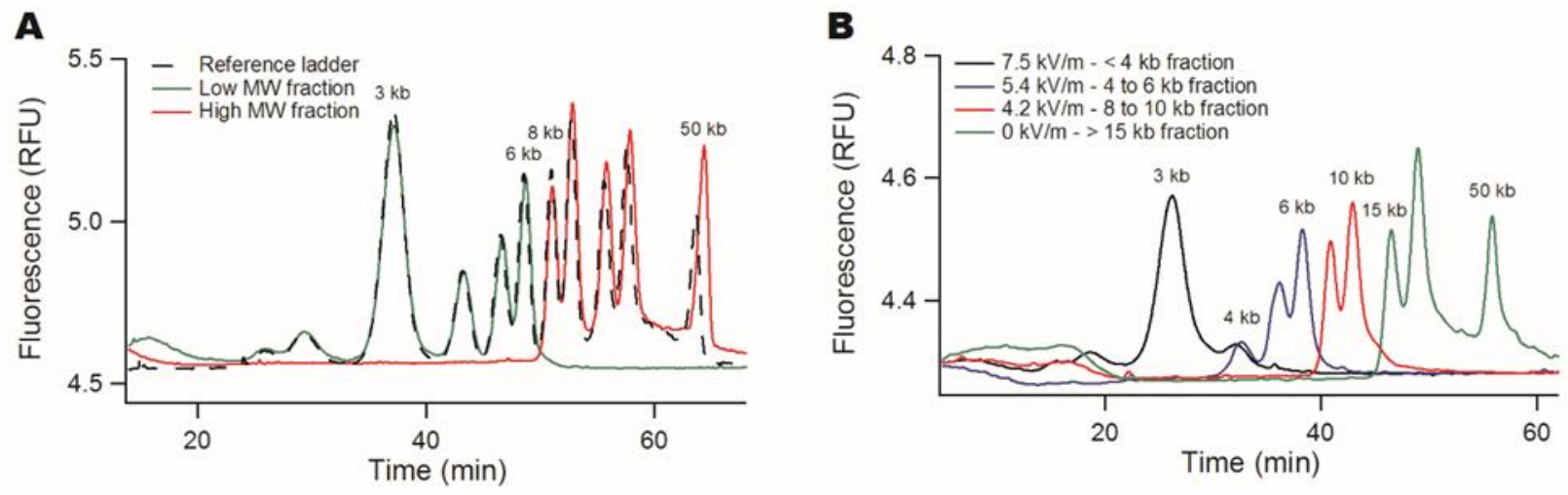

Figure 2: Tunable DNA size filtration in a monocapillary. (A) The chromatograms show the separation of the DNA ladder with the $\mu$ LAS technology using the separation settings reported in ref. ${ }^{28}$. The dashed black curve corresponds to the reference ladder with a few annotated bands, and the leak and retained fractions are plotted in green and red, respectively. The electric field was set to $5.4 \mathrm{kV} / \mathrm{m}$ during the selection phase, corresponding to a cut-off size of $\sim 7 \mathrm{~kb}$. The red and green curves have been multiplied by the dilution factors of 11.5 and 30, respectively. (B) The same ladder shown in (A) is fractionated into four fractions using three consecutive settings for the electric field of 7.5, 5.4, 4.2 kV/m, and a final collection step without any electric field (as indicated in the legend).

During the second step, we collected the retained high MW fraction by hydrodynamics only during ten minutes in a fresh vial containing $10 \mu \mathrm{L}$ of buffer. The final volume of the collected sample was $11.5 \mu \mathrm{L}$. The leak and retained fractions were subsequently analyzed using the $\mu$ LAS high MW separation method developed in ref. ${ }^{28}$ in order to determine their molecular composition and concentration. In Fig. $2 \mathrm{~A}$, we report the reference ladder together with the leak and retained fractions (black, green, and red curves, respectively), as obtained with a selection filter actuated with an electric field of $5.4 \mathrm{kV} / \mathrm{m}$. The size of the cut-off appeared to be $\sim 7 \mathrm{kbp}$ with a sharp separation between the bands of 6 and $8 \mathrm{kbp}$. The superposition of the different curves testified that the yield of this purification process was higher than $\sim 80 \%$ for all the bands in the sample.

We then modulated the electric field in order to fractionate the same ladder sample into four fractions. Using three threshold voltages of 7.5, 5.4, and $4.2 \mathrm{kV} / \mathrm{m}$, we performed three consecutive purification phases of one hour each, and finally collected the remaining fraction during 10 minutes. The resulting four fractions were analyzed to evaluate the three size selection cut-offs of 4, 7 and $12 \mathrm{kbp}$ (Fig. 2B). The final retained fraction plotted in green contained the peaks of 15,20 , and $50 \mathrm{kbp}$, suggesting that our technology was adequate for high MW DNA purification in the context of TGS technologies. Furthermore, the size selection threshold could 
be adjusted to $\sim 40 \mathrm{kbp}$, i.e. with the purification of the $50 \mathrm{kbp}$ band from the rest of the ladder (Supplementary Fig. S2), by setting the electric field to $3 \mathrm{kV} / \mathrm{m}$.

We then checked the validity of our model to predict the size selection cut-off (Eq. (4)). We performed a series of 13 experiments using different settings for the flow velocity and the electric field in the range 0.3 to $1.5 \mathrm{~mm} / \mathrm{s}$ and 3 to $7 \mathrm{kV} / \mathrm{m}$, respectively. We estimated the cut-off size $N_{c}$ with a precision determined by the size difference between the last peak in the leak fraction and first peak in the retained fraction. We plotted $N_{c}$ as a function of the electric field (Fig. 3A), showing a non-linear decrease as the electric field increased. We also noted that $N_{c}$ increased with the flow velocity at constant electric field, in agreement with the prediction of our model in Eq. (4). This relevance of our model was strongly confirmed by plotting the size cut-off as a function of $V_{0} / E^{3}$ (Fig. 3B), because a linear trend associated to a Pearson coefficient $\mathrm{R}^{2}$ of 0.94 was detected (dashed line in Fig. 3B). Altogether, we demonstrate the principle of a versatile and predictable DNA selection filter with a tunable cut-off size in the range of 3 to $40 \mathrm{kbp}$ controlled by an electric field delivered by a commercial capillary electrophoresis system.

A

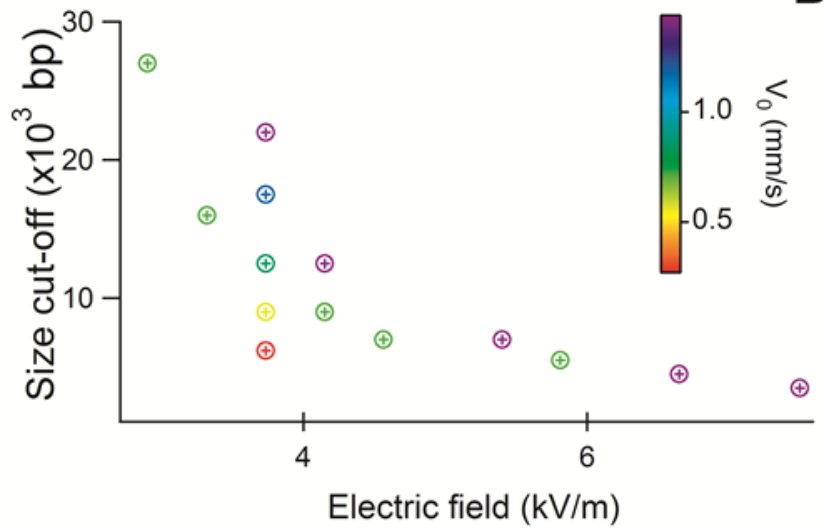

B

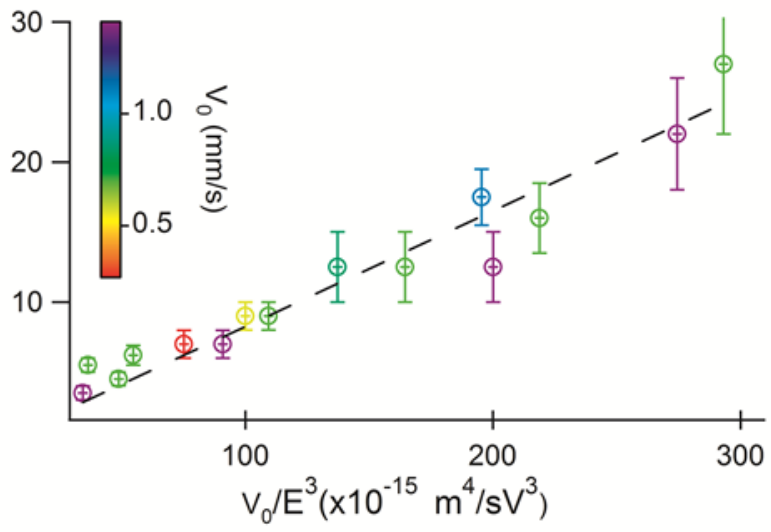

Figure 3: Control of the size selection threshold by the electric field. (A) The graph presents the size selection cut-off as a function of the electric field for various flow velocities, as indicated by the colorbar. Note that five experiments were carried out with a constant electric field of $3.8 \mathrm{kV} / \mathrm{m}$ and different flow velocities. (B) The same dataset can be cast on a master curve (dashed line) using the normalization suggested by our model (Eq. (4)).

\section{DNA saturation in the monocapillary device}

The production of 10 to $20 \mathrm{ng}$ of DNA is required for the standard work flow of linked-read sequencing. We thus aimed to evaluate the maximal amount of DNA that could be processed in the monocapillary device. 
We performed a series of experiments that consisted in injecting the DNA ladder in gradual increments starting from $0.5 \mathrm{ng}$ (i.e. five times more than in Fig. 2A) using a size selection cut-off at $9 \mathrm{kbp}$. Good size selection performances were obtained for an injection of $0.5 \mathrm{ng}$, as shown by the sharp separation between the curves representing the low MW leak and high MW retained fractions (dashed and solid lines in Fig. 4A, respectively). Note that the collection yield is higher than $90 \%$ for the bands of 10, 15, 20 and $50 \mathrm{kbp}$ (black solid line). As we doubled the amount of DNA, we noticed that the performances of the size selection filter were reduced because the bands of more than $10 \mathrm{kbp}$ were present in the leak fraction (dashed blue dataset in Fig. 4A). We concomitantly noticed that the collection yield decreased to $\sim 50 \%$. These trends were further enhanced using $1.5 \mathrm{ng}$ of DNA (red dataset in Fig. 4A). Because the bands of 10 to $50 \mathrm{kbp}$ corresponded to $34 \%$ of the whole ladder mass according to the supplier, we concluded that the monocapillary device was an efficient size selection filter for up to $\sim 0.2 \mathrm{ng}$ of DNA.

In order to investigate the saturation of the size selection filter, we performed live fluorescence microscopy analysis of the dynamics of DNA concentration at the constriction. We injected $8 \mathrm{ng}$ of $50 \mathrm{kbp}$ DNA, i.e. forty times more than the saturation threshold, and set the cut-off to $10 \mathrm{~kb}$ with an electric field and hydrodynamic flow velocity of $5.4 \mathrm{kV} / \mathrm{m}$ and $1.5 \mathrm{~mm} / \mathrm{s}$, respectively. We detected the accumulation of DNA molecules in the narrow capillary, and the formation of bright clusters of heterogeneous sizes (Fig. 4B). These clusters could not be stably retained, as shown by one escape event associated to hydrodynamic-dominated transport of a bright mass of DNA along the narrow channel (Fig. 4B). By approximating the region where DNA accumulates by a cylinder of $50 \mu \mathrm{m}$ in diameter and $200 \mu \mathrm{m}$ in height, we evaluated its volume to $\sim 0.4$ $\mathrm{nL}$. Taking the saturation threshold to be $0.2 \mathrm{ng}$, we then deduced that the size selection filter saturated for a DNA concentration of $\sim 0.5 \mathrm{mg} / \mathrm{mL}$. Note that this estimate is likely under evaluated because DNA accumulates close to the walls and not evenly across the capillary section. DNA concentration at saturation then appears to be lower than the solubility limit of this biomolecule in the range $10-100 \mathrm{mg} / \mathrm{mL}^{30}$, but larger than the threshold of DNA aggregation driven by AC electric fields of $0.05 \mathrm{mg} / \mathrm{mL}^{31}$. Consequently, irrespective to the mechanism of clustering, the saturation of the size selection filter appears to arise from the confinement of the retained fraction in a narrow volume and the ensuing molecular aggregation. 
A

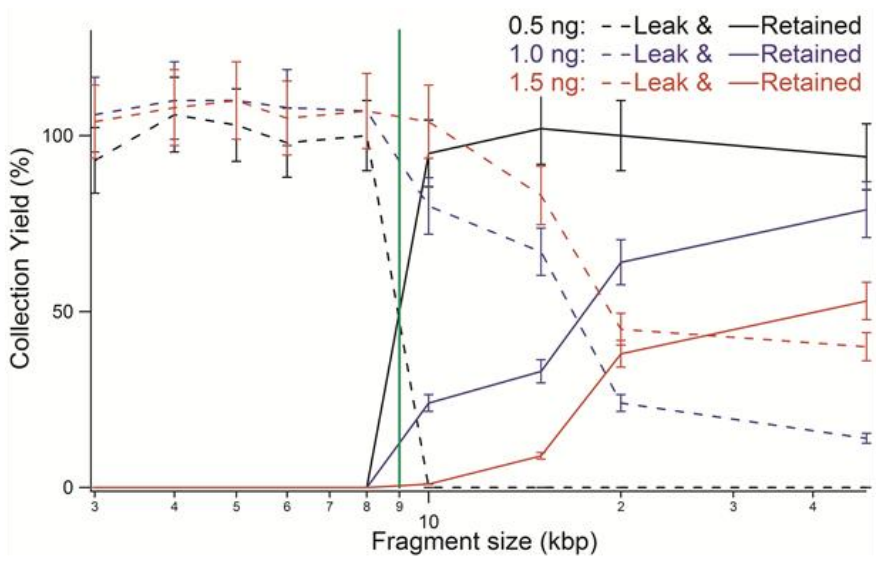

B
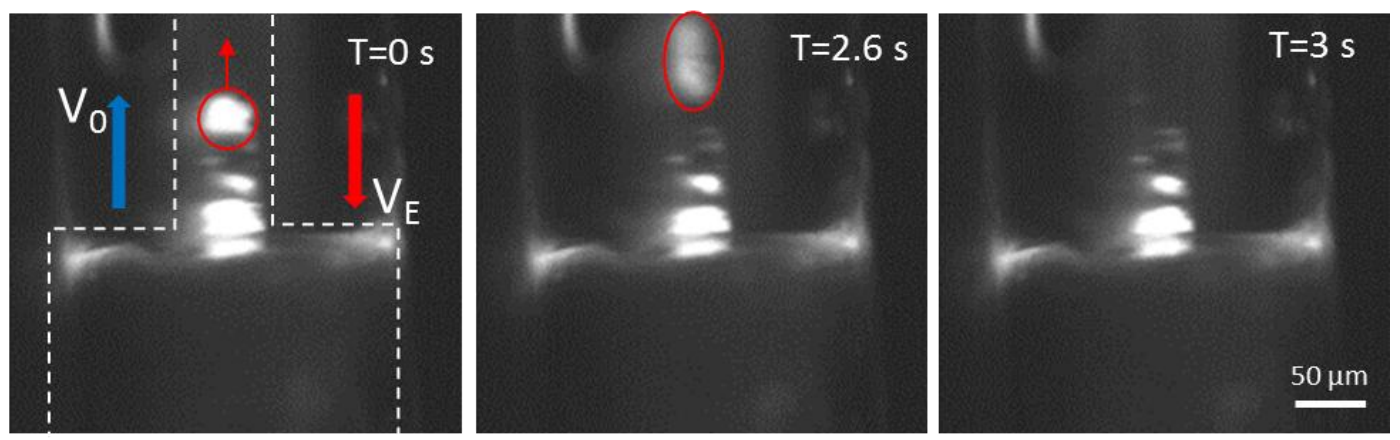

Figure 4: Saturation of the DNA filter. (A) The graph shows the yield of the leak and retained fractions (represented as dashed and solid lines, respectively) as a function of DNA size in logarithmic scale. We use the same ladder as in Fig. 2 and set the cut-off to $9 \mathrm{kbp}$ (green line). The concentration is determined with an error of 10\%, as shown in ref. ${ }^{28}$. (B) The

fluorescence micrographs present a time-lapse recording of the response of high MW DNA during the size selection process. Molecules are concentrated at the entry of the narrow capillary (shown with dashed white contour lines). The bright cluster marked in red is not retained and leaks away from the constriction.

\section{Scale up of the technology with multicapillary systems}

In order to increase the throughput of the size selection filter, we opted for the parallelization of technology and fabricated a multicapillary system containing 61 channels of $46 \mu \mathrm{m}$ in diameter (Fig. 1C). We indeed expected the saturation threshold to be multiplied by a factor of 60 , allowing $\sim 60 \times 0.2=12 \mathrm{ng}$ of DNA to be processed in one operation. Because the multicapillary system could not be integrated in commercial Capillary Electrophoresis instruments, we designed a prototype for sample fractionation (Supplementary Fig. S3). The gravitational flow was sufficient to induce a fluid velocity of $1.0 \mathrm{~mm} / \mathrm{s}$ in the channels (see Supplementary Material), thereby setting accurate actuation conditions for size filtration. Note 
that the size selection process was accelerated to 30 minutes because the length of the multicapillary was halved in comparison to the monocapillary.

We first checked that the size selection threshold was similar by performing sample fractionation with an electric field set to $4 \mathrm{kV} / \mathrm{m}$ during the retention phase. We expected a size cut-off of $12 \mathrm{~kb}$, and measured it at 9 kbp (Supplementary Fig. S4A), confirming that the operating principle of the technology remained nearly the same in the mono- and multi-capillary systems. The quality of the selection filter's cut-off was slightly decreased, as we detected residual amounts of the 8 and $6 \mathrm{kbp}$ bands in the retained fraction of 7 and $3 \%$, respectively. In order to select high MW DNA for sequencing applications, we then established the size selection cut-off to $\sim 40 \mathrm{kbp}$ by adjusting the electric field to $2.9 \mathrm{kV} / \mathrm{m}$ (Fig. 5). For this experiment, we spiked the DNA ladder with an additional DNA fragment of $100 \mathrm{kbp}$, which was collected in the retained fraction together with the $50 \mathrm{kbp}$ band (red curve in Fig. 5). Because the threshold was close to $50 \mathrm{kbp}$, we also detected a small proportion of $50 \mathrm{kbp}$ DNA in the leak fraction (blue curve in Fig. 5). These experiments hence showed that the selection technology could be operated with a multicapillary system with the same electrohydrodynamic actuation parameters as in the monocapillary.

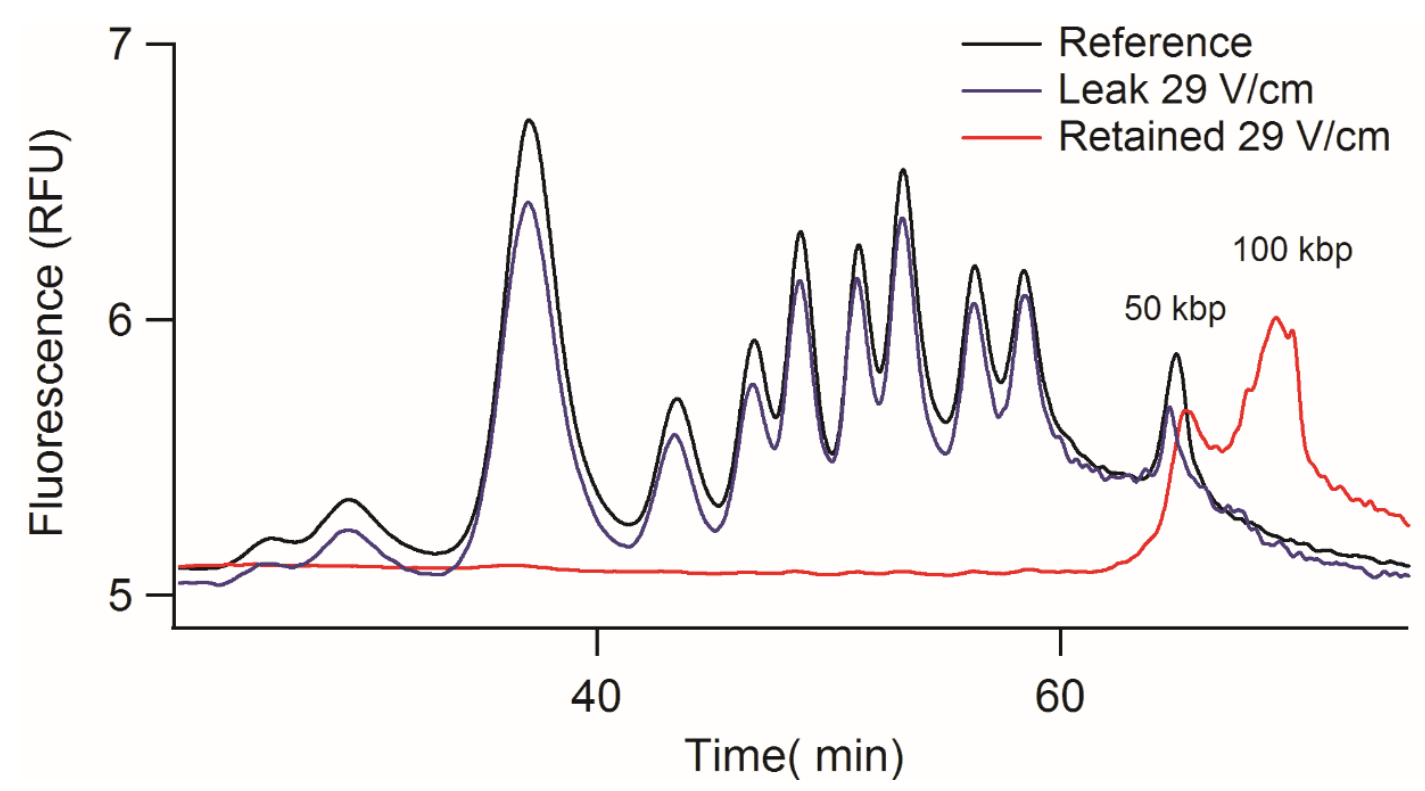

Figure 5: High MW size selection in the multicapillary system. The plot presents chromatograms with the reference ladder with bands of 3 to $50 \mathrm{kbp}$ (dashed black curve), and the leak and retained fractions in blue and red, respectively. The electric field was set to $2.9 \mathrm{kV} / \mathrm{m}$ during the selection phase. The red and blue curves have been multiplied by the dilution factors 60 and 260 , respectively. 
We then evaluated the saturation threshold of the multicapillary device by injecting gradual amounts of the $\mathrm{kb}$ extend DNA ladder from 5 to $50 \mathrm{ng}$, i.e. $1.5 \mathrm{ng}$ to $15 \mathrm{ng}$ retained at the constriction, with the same actuation parameters as in Supplementary Fig. S4A. The collection yield appeared to be lower in the multicapillary vs. monocapillary system. We indeed measured collection yields of $75 \%$ and $55 \%$ for the leak and retained fractions, respectively (Supplementary Fig. S4B). This apparent loss of DNA remains unclear, but we suspect that some molecules remain trapped on the outer glass shell of the multicapillary of $250 \mu \mathrm{m}$ (Fig. 1C) where the flow velocity and electric fields are nearly null. The selection cut-off appeared to be sharp for 1.5 and $4.5 \mathrm{ng}$ of high MW DNA at the constriction, but the size selection cut-off broadened for $15 \mathrm{ng}$ of DNA. The presence of the 10 to $50 \mathrm{kbp}$ fragments in the leak fraction indicated the saturation of the multicapillary system, likely associated to the formation of aggregates during the size selection process. Consequently, the scale up of our technology to 61 capillaries allowed us to increase the saturation threshold by a factor of 4/0.2 20, meeting the objectives of increasing the throughput without changing the actuation settings.

\section{High MW DNA selection for long read sequencing}

We finally proceeded to the selection of high MW genomic DNA from the melon Cucumis melo L., an important horticultural crop worldwide with a diploid genome of $\sim 450 \mathrm{Mbp}^{32}$. The Melon genomic DNA was purified in liquid phase, resulting in a broad size distribution from 2 to more than $50 \mathrm{kbp}$ (black curve in Fig. 6A), as confirmed by FEMTO Pulse analysis (Supplementary Fig. S5). The presence of low MW residues justified the process of size selection for TGS. Our experiments hence consisted in assessing the quality of sequencing data obtained with or without size selection. They were carried out by the construction of a DNA library with the gel bead partitioning system, called the Chromium technology, followed by sequencing on an Illumina SGS platform (See Methods).

We first processed a low quantity of $2.5 \mathrm{ng}$ of the Melon DNA sample using the same voltage threshold of $2.9 \mathrm{kV} / \mathrm{m}$ as for the DNA ladder. As expected, the retained fraction was larger than $\sim 40 \mathrm{kbp}$ (red curve in Fig. 6A) and the leak fraction was centered at $\sim 25 \mathrm{kbp}$ (blue curve in Fig. 6A). Next, we aimed to increase the collection to reach the $\sim 15 \mathrm{ng}$ required for sequencing operations. Because the proportion of molecules of more than $40 \mathrm{kbp}$ represented $\sim 20 \%$ of the sample and the collection yield was $\sim 50 \%$, we performed the operation with an initial input of $100 \mathrm{ng}$. In addition, we repeated the size selection operation 4 times consecutively so as 
to retain $5 \mathrm{ng}$ at the constriction each time and avoid saturation. At the end of this selection process, which took place in 2 hour, we collected $25 \mu \mathrm{L}$ of material at a concentration of $0.7 \mathrm{ng} / \mu \mathrm{L}$. Hence, we obtained $\sim 17 \mathrm{ng}$ of genomic DNA, in good agreement with our initial specifications. The size distribution of the purified sample was not as sharp as in the calibration experiment, as shown by the presence of molecules of $15 \mathrm{~kb}$ (red curve in Fig. 6B), but the removal of DNA fragments lower than $\sim 10 \mathrm{kbp}$ was clearly achieved. Because the selection threshold was lower than expected, we note that the recovery yield was lower than initially expected. This degradation of size selection performances may be due to the rapid saturation of the filter due to the presence of very high MW molecules (see more below). We indeed noticed that saturation was decreasing with DNA MW, as for instance exemplified by the saturation at $5 \mathrm{ng}$ with a fragment of $0.2 \mathrm{kbp}$ (data not shown).

A

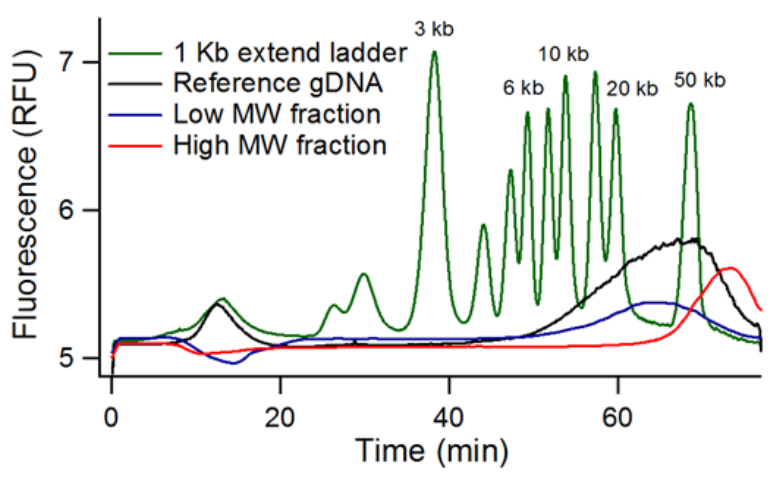

B

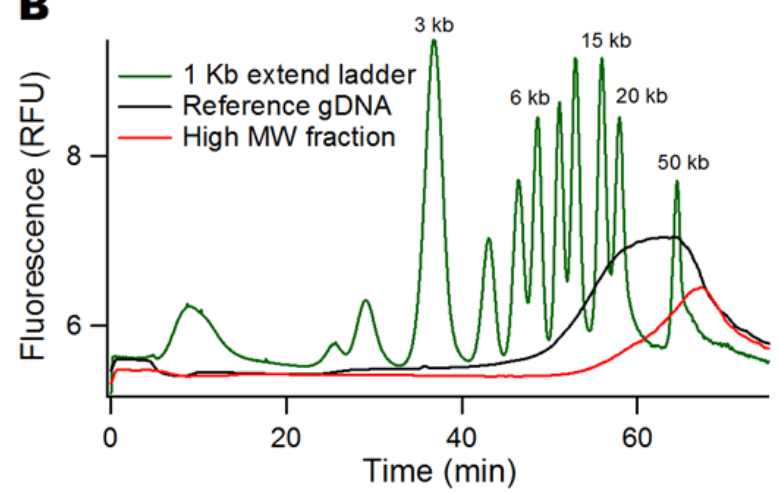

Figure 6: High MW size selection of genomic DNA. (A) The graph shows the DNA ladder in green, the unpurified melon genomic DNA sample in black, and the outputs of the size selection process on the multicapillary system in blue and red. The threshold electric field is set to $2.9 \mathrm{kV} / \mathrm{m}$ for a threshold size of $\sim 40 \mathrm{kbp}$. This calibration experiment is carried out with $2.5 \mathrm{ng}$ of genomic DNA. (B) The chromatogram shows the same size selection process carried out four times with $100 \mathrm{ng}$ of DNA input in order to collect $15 \mathrm{ng}$ of high MW DNA.

We sequenced processed vs. unprocessed genomic DNA samples, and obtained the same assembly size of $\sim 366 \mathrm{Mbp}$, representing $\sim 81 \%$ of the genome (Table 1). This size range was comparable to that obtained by pyrosequencing of $375 \mathrm{Mbp}^{32}$. The average length of the sequenced DNA fragments was also comparable in both samples (second line in Table 1), in apparent contradiction with the size selection process. Conversely, the number of contigs longer than $50 \mathrm{kbp}$ was $63 \%$ greater after size selection by $\mu$ LAS. These results can be explained if some high MW molecules are eliminated during the selection process, probably because they form clusters and tend to leak during the selection phase. The sequencing of these long molecules increases the 
coverage of the genome, explaining that the scaffold N50 size was 68\% longer without purification. Nevertheless, the narrowing of the size distribution after size selection (red curve in Fig. 6B) allows us to obtain a higher number of contigs of more than $50 \mathrm{kbp}$. Interestingly, during library preparation, the partitioning of the unprocessed sample with its broad size distribution creates discrepancies in the depth of coverage $^{33}$, likely resulting in low quality sequencing data for long molecules. Contrariwise, the narrow size distribution of DNA fragments obtained with $\mu$ LAS insures homogeneous depth of sequencing and quality data for the identification of single nucleotide polymorphisms. This proposition explains the doubling of the N50 phase block size, which scores the accurate representation of the two chromosomes from sequencing data, after size selection as well as the better performances in terms of average number of uncalled bases (lower line in Table 2). Altogether, because the resolution of haplotypes is a key asset of Chromium technology, we conclude that the size selection of genomic DNA with $\mu \mathrm{LAS}$ is not indispensable for sequencing but enables us to obtain quality sequencing data.

\begin{tabular}{|c|c|c|}
\hline & Without size selection & $\mu$ LAS \\
\hline Assembled size (Mbp) & 367 & 366 \\
\hline Length-weighted mean (kbp) & 25.0 & 24.6 \\
\hline Contig > 50 kbp & 258 & 420 \\
\hline Largest Contig (Mbp) & 6.8 & 7.2 \\
\hline Scaffold N50 (Mbp) & 2.58 & 1.54 \\
\hline N50 phase block (Mbp) & 8.1 & 16.5 \\
\hline Average number of uncalled & 3.0 & 2.5 \\
bases per kbp & & \\
\hline
\end{tabular}

Table 1: Metrics of the sequencing data obtained by the Chromium technology.

\section{Discussion}

We describe a size-accordable filter for high MW DNA selection. On a monocapillary system, the sharp size selection threshold can be adjusted in the range of 3 to $40 \mathrm{kbp}$ to perform sample fractionation with collection yields higher than $90 \%$. We show that the filter saturates if more than $\sim 0.2$ ng of DNA is processed, 
and demonstrate that saturation is associated to an aggregation mechanism. In order to produce genomic material for linked-read sequencing, we scale up the technology with a multicapillary system, and report the collection of high MW DNA from a Melon genomic DNA sample using a process of two hour. The resulting material is sequenced, and sequencing data appear to be improved for diploid genome resolution with size selection $v s$. without it.

Future lines of development concern the better processing of DNA molecules of more than $\sim 100 \mathrm{kbp}$. Our data indeed indicate that these molecules tend to be eliminated during size selection. Consistently, this limitation has not been detected during the calibration steps, which have been performed with a DNA ladder with a larger band of $100 \mathrm{kbp}$. Hence, specific improvements should be performed to define operating conditions in the range of 50 to $300 \mathrm{kbp}$ with a dedicated ladder. While we recently showed that DNA separation could be performed for fragments of up to $200 \mathrm{kbp}^{28}$, the saturation of our technology remains to be evaluated in this size range. In the low MW size limit, size selection would also be valuable for other applications, including enhanced analysis of circulating cell-free DNA in blood plasma ${ }^{34}$. The adequate formulation of the viscoelastic buffer has already been reported for separation and concentration for low MW DNA $^{19}$, and promising results of purification have been obtained with a favorable saturation threshold of $5 \mathrm{ng}$ in a monocapillary system (data not shown).

Regarding future applications, the potential of $\mu \mathrm{LAS}$ for purification of minute amounts of genomic material and its sensitivity of $10 \mathrm{fg} / \mu \mathrm{L}^{28}$ may be particularly useful for single cell sequencing studies ${ }^{16}$. In this context, the number of molecules is minimal and the collection yield critical. Because one human cell contains a few pg of DNA, saturation is not expected to be an issue. Hence, purification operations may be performed in the monocapillary system, which shows best performances. The preservation of long chromosome fragment integrity throughout the size selection process should carefully be evaluated, requiring the development of specific solutions for quality control of minute samples of very high MW. The resulting technologies for quality control and size selection may contribute to the better analysis of genomic heterogeneities and the interplay between allele variation and gene expression. 
Acknowledgements. We are thankful to Xavier Dollat for the manufacturing of the multicapillary system. This project was partly supported by the project "Gene Extractor" from Région Midi-Pyrénées and by the ANR $\mu$ LAS (ANR-16-CE18-0028-01) and ANR BioPulse (ANR-16-ASTR-0020).

\section{References}

1 M. J. Chaisson, J. Huddleston, M. Y. Dennis, P. H. Sudmant, M. Malig, F. Hormozdiari, F. Antonacci, U. Surti, R. Sandstrom and M. Boitano, Nature, 2015, 517, 608.

2 J. Huddleston, S. Ranade, M. Malig, F. Antonacci, M. Chaisson, L. Hon, P. H. Sudmant, T. A. Graves, C. Alkan and M. Y. Dennis, Genome research, 2014, 24, 688-696.

3 L. Gong, C.-H. Wong, W.-C. Cheng, H. Tjong, F. Menghi, C. Y. Ngan, E. T. Liu and C.-L. Wei, Nat Methods, 2018, 15, 455-460.

4 M. C. Stancu, M. J. Van Roosmalen, I. Renkens, M. M. Nieboer, S. Middelkamp, J. De Ligt, G. Pregno, D. Giachino, G. Mandrile and J. E. Valle-Inclan, Nature communications, 2017, 8, 1326.

5 A. L. Norris, R. E. Workman, Y. Fan, J. R. Eshleman and W. Timp, Cancer biology \& therapy, 2016, 17, 246-253.

6 E. L. van Dijk, Y. Jaszczyszyn, D. Naquin and C. Thermes, Trends in Genetics, 2018, 34, 666-681.

7 J. M. Feehan, K. E. Scheibel, S. Bourras, W. Underwood, B. Keller and S. C. Somerville, JoVE (Journal of Visualized Experiments), 2017, e55463.

8 R. Boom, C. J. Sol, M. M. Salimans, C. L. Jansen, P. M. Wertheim-van Dillen and J. van der Noordaa, J. Clin. Microbiol., 1990, 28, 495-503.

9 L. Becker, M. Steglich, S. Fuchs, G. Werner and U. Nübel, Scientific reports, 2016, 6, 28063.

10 B. Mayjonade, J. Gouzy, C. Donnadieu, N. Pouilly, W. Marande, C. Callot, N. Langlade and S. Muños, BioTechniques, 2016, 61, 203-205.

11 M. A. Quail, Y. Gu, H. Swerdlow and M. Mayho, Electrophoresis, 2012, 33, 3521-3528.

12 E. L. Van Dijk, Y. Jaszczyszyn and C. Thermes, Experimental cell research, 2014, 322, 12-20.

13 A.-C. Portmann, C. Fournier, J. Gimonet, C. Ngom-Bru, C. Barretto and L. Baert, Frontiers in microbiology, 2018, 9, 446.

14 J. Stadler, R. Lemmens and T. Nyhammar, J Gene Med, 2004, 6 Suppl 1, S54-66.

15 M. H. Tan, C. M. Austin, M. P. Hammer, Y. P. Lee, L. J. Croft and H. M. Gan, GigaScience, 2018, 7, gix137.

16 E. Shapiro, T. Biezuner and S. Linnarsson, Nature Reviews Genetics, 2013, 14, 618.

17 L. R. Huang, J. O. Tegenfeldt, J. J. Kraeft, J. C. Sturm, R. H. Austin and E. C. Cox, Nat Biotech, 2002, 20, $1048-1051$.

18 B. H. Wunsch, S.-C. Kim, S. M. Gifford, Y. Astier, C. Wang, R. L. Bruce, J. V. Patel, E. A. Duch, S. Dawes and G. Stolovitzky, Lab on a Chip, 2019, 19, 1567-1578.

19 C.-L. Andriamanampisoa, A. Bancaud, A. Boutonnet-Rodat, A. Didelot, J. Fabre, F. Fina, F. Garlan, S. Garrigou, C. Gaudy and F. Ginot, Analytical chemistry, 2018, 90, 3766-3774.

20 N. Milon, C. Chantry-Darmon, C. Satge, M.-A. Fustier, S. Cauet, S. Moreau, C. Callot, A. Bellec, T. Gabrieli and L. Saïas, Nucleic acids research, 2019, 47, 8050-8060.

21 H. Ranchon, R. Malbec, V. Picot, A. Boutonnet, P. Terrapanich, P. Joseph, T. Leïchlé and A. Bancaud, Lab Chip, 2016, 16, 1243-1253.

22 R. Malbec, B. Chami, L. Aeschbach, G. A. R. Buendía, M. Socol, P. Joseph, T. Leïchlé, E. Trofimenko, A. Bancaud and V. Dion, Scientific reports, 2019, 9, 23.

23 P. Marks, S. Garcia, A. M. Barrio, K. Belhocine, J. Bernate, R. Bharadwaj, K. Bjornson, C. Catalanotti, J. Delaney and A. Fehr, Genome research, 2019, 29, 635-645.

24 B. Chami, M. Socol, M. Manghi and A. Bancaud, Soft matter, 2018, 14, 5069-5079.

25 M. Socol, H. Ranchon, B. Chami, A. Lesage, J.-M. Victor, M. Manghi and A. Bancaud, Macromolecules, 2019, 52, 1843-1852.

26 M. Arca, A. J. Ladd and J. E. Butler, Soft matter, 2016, 12, 6975-6984.

27 J. E. Butler, O. B. Usta, R. Kekre and A. J. C. Ladd, Physics of Fluids, 2007, 19, 113101. 
28 N. Milon, C. Chantry-Darmon, C. Satge, M. A. Fustier, S. Cauet, S. Moreau, C. Callot, A. Bellec, T. Gabrieli, L. Saias, A. Boutonnet, F. Ginot, H. Bergès and A. Bancaud, Nucl Acids Res.

29 P. Russell, science, 2003, 299, 358-362.

30 J. E. Cleaver and H. W. Boyer, Biochimica et Biophysica Acta (BBA)-Nucleic Acids and Protein Synthesis, 1972, 262, 116-124.

31 L. Mitnik, C. Heller, J. Prost and J. L. Viovy, Science, 1995, 267, 219-222.

32 J. Garcia-Mas, A. Benjak, W. Sanseverino, M. Bourgeois, G. Mir, V. M. González, E. Hénaff, F. Câmara, L. Cozzuto and E. Lowy, Proceedings of the National Academy of Sciences, 2012, 109, 11872-11877.

33 N. I. Weisenfeld, V. Kumar, P. Shah, D. M. Church and D. B. Jaffe, Genome Res, 2017, 757-767.

34 F. Mouliere, D. Chandrananda, A. M. Piskorz, E. K. Moore, J. Morris, L. B. Ahlborn, R. Mair, T. Goranova, F. Marass and K. Heider, Science translational medicine, 2018, 10, eaat4921.

\section{Table TOC}

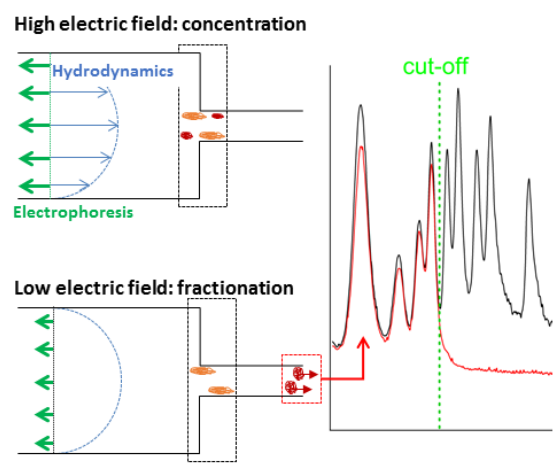

